\title{
FORMALIZACIÓN DE LA TRANSMISIÓN DEL CUIDADO DURANTE LA ETAPA VITAL INTRAUTERINA
}

\section{FORMALIZATION OF CARE TRANSMISSION DURING THE INTRAUTERINE VITAL STAGE}

\author{
Zaida Pérez Chico
}

Hospital Universitario Príncipe de Asturias (SERMAS) 


\section{Resumen}

La forma en la que la mujer lleva a cabo sus cuidados durante el embarazo es de gran importancia para el desarrollo prenatal del feto, el cual tiene lugar durante la etapa vital intrauterina.

Este cuidado materno tendrá una repercusión en el feto que se manifestarán antes, durante o después del nacimiento en función de la etapa del desarrollo prenatal en el que hayan tenido lugar.

Puesto que el feto es sujeto de cuidado dependiente $y$, la responsable de su autocuidado es la madre durante la gestación, se consideró necesario hacer un estudio que formalice la repercusión fetal del autocuidado materno.

Para ello se aplica metodología del tipo deductiva con el fin de demostrar que dicha relación existe (autocuidado materno - repercusión en el feto).

Palabras claves: Autocuidado, desarrollo prenatal, Modelo de Autocuidado de Orem, herencia

\section{Abstract}

The way in which the woman carries out her care during pregnancy is of great importance for the prenatal development of the fetus, which takes place during the intrauterine life stage.

This maternal care will have an impact on the fetus that will manifest before, during or after birth depending on the stage of prenatal development in that has taken place.

Since the fetus is the subject of dependent care and the one responsible for its self-care is the mother during pregnancy, it is considered necessary to carry out a study that formalizes the fetal repercussion of maternal self-care.

For this, deductive type methodology is applied in order to demonstrate that such relationship exists (maternal self-care - impact on the fetus).

Key words: Self care, prenatal development, Orem Self-care Model, Genetics 


\section{INTRODUCCIÓN}

El embarazo es un proceso vital de suma importancia que provoca cambios en las necesidades y competencias de la madre, de tal manera, que ésta deberá asumir nuevas prácticas de cuidados y/o adaptar los ya conocidos. (17)

Durante este proceso, los cuidados maternos, tendrán repercusión sobre el feto que se desarrolla dentro del útero materno, quién, durante esta etapa del desarrollo será dependiente de sus cuidados.

Actualmente y, a pesar de que la información está al alcance de la mayoría de la población, siguen naciendo niños que presentan anomalías congénitas o determinadas patologías, como el Síndrome de Abstinencia Neonatal, Síndrome Alcohólico Fetal... como consecuencia de unos cuidados inadecuados durante esta etapa del desarrollo.

Como profesionales de Enfermería, cuya función principal es la prestación de cuidados, considero necesario analizar el cuidado materno a través de un Marco Teórico enfermero con el fin de formalizar la repercusión que tienen éstos sobre el feto durante la etapa vital intrauterina, ya que no existen estudios de este tipo.

Para ello será necesario el estudio de los elementos del Metaparadigma de Enfermería: persona y cuidado, así como, el estudio de la Herencia humana y la etapa vital Intrauterina. 


\section{ESTADO DEL ARTE}

\section{Descripción de persona}

Comenzaremos definiendo persona como "individuo de la especie humana," (1) lo que la confiere como "hombre, ser humano" y, a su vez, como "ser vivo" (1), con capacidad de nacer, crecer y reproducirse, y por lo tanto generar "material semejante al que les compone" y transmitirlo a sus descendientes. (2)

Además, el ser humano, en su condición de mamífero, por definición es aquel que "alberga a sus crías en el seno materno". (1) Esto tiene lugar en la primera etapa del desarrollo humano, es decir en la etapa prenatal, que es aquella que va desde la concepción hasta el nacimiento. Se divide en periodo embrionario (de la fertilización hasta el final de la octava semana) y periodo fetal (de la novena semana al nacimiento). Estos periodos son fundamentales, pues en ellos tendrá lugar la etapa de organogénesis y la posterior maduración en la que los aparatos y sistemas "aprenderán" a funcionar hasta ser capaces de hacerlo de manera independiente. (3)

\section{Existen determinados factores} (internos o externos), capaces de atravesar la barrera placentaria y provocar afectación fetal, denominados factores teratogénicos, por lo que diremos que un teratógeno es "cualquier agente que cause una anomalía congénita tras la exposición fetal durante el embarazo". (4) Su efecto dependerá de la duración, tiempo de exposición, salud materna y etapa del desarrollo (general y de cada órgano). (4) (5)

Continuando con la conceptualización de persona, a lo largo de la historia, los filósofos se han preguntado por el significado del ser humano. Con el fin de conocer la evolución de la conceptualización del mismo a lo largo de la historia, realizaremos una breve descripción del significado del ser humano para los principales filósofos desde la Antigüedad griega hasta el S. XX.

Con Platón tenemos un cambio en la concepción del hombre como ser de estructura dual. Este dualismo ontológico nos lleva a una visión del ser humano que para Platón estaría compuesto de cuerpo y alma. (6)

A diferencia de Platón, Aristóteles afirma que el ser humano es un constructo hilemórfico, es decir, un compuesto de Materia y Forma; sostiene que no somos una unión accidental, sino natural y necesaria. Además, el ser humano tiende a la Eudaimonía (felicidad); ésta debe basarse en la naturaleza humana y debe partir de los hechos de la experiencia personal. Para Aristóteles, la felicidad se identifica con la autorrealización, la racionalidad y la virtud. Debe ser algo práctico y humano. (7) 
Para Kant, el ser humano como "ser dotado de razón y voluntad libre, es un fin en sí mismo, que a su vez puede proponerse fines." (8)

Kant lleva a cabo un análisis de la razón, de sus capacidades y de sus límites. Para ello, establece que existen juicios a priori, que se encuentran al margen de la experiencia y son universales y necesarios, a la par que juicios a posteriori, frutos de la experiencia. Para Kant "todo conocimiento empieza con la experiencia, pero no todo conocimiento proviene de la experiencia". (6)

Para Heidegger la tarea de la filosofía era reflexionar sobre el ser, el Dasein. Desde el punto de vista de la fenomenología analiza la estructura constitutiva del ser humano dejando que los fenómenos se muestran en su forma original. (6)

Sin embargo, Heidegger no entiende la fenomenología sin la Hermenéutica ya que, para él, el sujeto no se va a acercar a la realidad de una forma "pura", considera que siempre va a haber una interpretación por al menos dos motivos: por un lado, estará basada en sus experiencias, y por otra parte considera que el observador tampoco es puro.

Esto quiere decir para Heidegger que un fenómeno puede ser visto de diferentes maneras, dependiendo de cómo se tenga acceso a él o en qué momento sea visto. (9)

En cuanto a la conceptualización de persona desde el Marco conceptual de Enfermería, abordaremos la realizada por D. Orem que define a la persona como "una unidad con funciones biológicas, simbólicas y sociales, y centra su atención en la capacidad de éstas de para transformar su autocuidado." (10)

La idea de persona como agente es básica en el desarrollo de su teoría, definida como "persona que emprende una acción o tiene la facultad para hacerla." (11) Esta capacidad de las personas para realizar acciones puede verse afectada por una serie de factores internos 0 externos, para ocuparse de su autocuidado, afectando tanto al tipo como a la calidad de autocuidado requerido, se denominan factores condicionantes básicos. (12)

Estos FCB son: edad, sexo, estado de desarrollo, estado de salud, orientación sociocultural, factores asociados al sistema de salud, factores asociados al sistema familiar, patrones o estilos de vida, factores ambientales, así como los recursos disponibles y la adecuación de los mismos. (11) 


\section{DESCRIPCIÓN DEL CUIDADO}

En primer lugar, para describir el cuidado cabe reseñar la definición propuesta por M. F. Collière: en su libro Promover la vida, que define cuidar como "un acto de mantener la vida asegurando la satisfacción de un conjunto de necesidades indispensables, pero que son diversas en su manifestación. Las diferentes posibilidades de responderá estas necesidades vitales crean e instauran hábitos de vida propios de cada grupo o persona." (13)

Además, se ha de tener en cuenta, tal y como describe el Dr. Santamaría en su libro "Los axiomas del cuidado" que "el Cuidado es energía" (14) y que además, esta energía de cuidado puede tener dirección de acción: de entrada o de salida, de tal manera que, los cuidados pueden ir dirigidos hacia uno mismo (Cuidar_me) o hacia el otro (Cuidar_te). Asumiendo este axioma, se puede inferir que los actos de cuidado de una mujer gestante irán dirigidos por un lado hacia sí misma (Cuidar_me) y, por otro lado, hacia el feto que se está desarrollando en su interior (Cuidar_te).

Se ha de tener en cuenta que, atendiendo a las investigaciones del Dr. Santamaría, estos cuidados puede ir dirigidos hacia la supervivencia o hacia el bienestar, encontrándonos así con las diferentes posibilidades del cuidado. (14)
Siguiendo con la conceptualización del cuidado, y como ya se ha mencionado anteriormente, abordaremos la obra estética "Los Axiomas del Cuidado" donde axioma, según la RAE, es una "proposición tan clara y evidente que se admite sin demostración" y, como "cada uno de los principios fundamentales e indemostrables sobre los que se construye una teoría". (1)

Por otro lado, Orem define el autocuidado como la "capacidad de actuar intencionadamente para regular los factores que afectan a su propio funcionamiento y desarrollo." (11) Reconoce tres tipos de requisitos de autocuidado:

- Requisitos de Autocuidado Universal: "comunes a todos los seres humanos durante todas las etapas de la vida, ajustadas a la edad, estado desarrollo, factores del entorno y otros." (11)

- Requisitos de Autocuidado del desarrollo: son acciones que necesitan ser realizadas en relación con los procesos de desarrollo humano, condiciones y acontecimientos, así como con los sucesos que pueden afectar negativamente al desarrollo. (10) (11)

- Requisitos de Autocuidado relativos a la desviación de la salud: estos requisitos existen para las personas que se encuentran 
en situación de enfermedad o sufren alguna lesión y para los individuos que están siendo sometidos a un diagnóstico y tratamiento médico. ${ }^{(10)}$

Define cuidado dependiente como la "práctica de actividades que personas responsables, maduras o en proceso de maduración inician o ejecutan en beneficio de personas socialmente dependientes durante cierto tiempo, o de manera continuada, para mantener su vida y contribuir a sus salud $y$ bienestar." (11)

Otro pilar importante de este estudio es la herencia humana, motivo por el cual procedo a describirlo en el siguiente punto.

\section{Descripción de HeREnCia}

La "Genética" es la "parte de la biología que trata de la herencia y de lo relacionado con ella." (1) La RAE define "Herencia" como el "conjunto de caracteres que los seres vivos reciben de sus progenitores," así como los "rasgos morales, científicos, ideológicos, etc., que, habiendo caracterizado a alguien, continúan advirtiéndose en sus descendientes" y "circunstancias de índole cultural, social" en su tercera, cuarta y quinta acepción respectivamente. (1)
Para tener una mejor comprensión de esta definición se conceptualizan el término caracteres, de tal manera que los caracteres son el "conjunto de cualidades o circunstancias propias de una cosa, de una persona o de una colectividad, que las distingue, de las demás," según la $6^{a}$ acepción de la RAE, (1) que incluye una serie de acepciones que se definen por su relevancia:

- Carácter adquirido: "Cada uno de los rasgos anatómicos o funcionales no heredados, sino adquiridos por el animal durante su vida." (1)

- Carácter heredado: "Cada uno de los rasgos anatómicos o funcionales que se transmiten de una generación a otra." (1)

El desarrollo del ser humano tiene un doble componente de información hereditaria, por un lado, la herencia biológica, constituida fundamentalmente por el genoma y, por otra parte, la herencia cultural que es "aquella que cada ser humano recibe de su entorno familiar y social, compuesta por información de tipo cultural de elementos aprendidos y que se encuentra disponible en forma de símbolos escritos o de prácticas tradicionales". (15)

Según Beorlegui, "el genoma nos aporta una serie de predisposiciones, no 
tanto constricciones y estructuras rígidas, y sobre ella actúan nuestras decisiones libres y la cultura en la que vivimos." (15)

Son varios los autores que hablan de "herencia dual." Distinguen la transmisión cultural de la transmisión genética, pero manifiestan la importancia de la interacción entre ellas. (16)

Por lo tanto, parece claro que cuando hablamos de herencia humana, la biología y la cultura son dos procesos unidos entre sí. De hecho, mientras que la especia humana siga existiendo y el medio ambiente, social y cultural, siga cambiando, existen razones para pensar que continuará produciéndose la selección natural de las características biológicas y culturales de las personas. (16)

\section{OBJetivos}

A continuación, procedo a detallar el objetivo principal de este estudio de investigación: describir la transmisión de la herencia del cuidado.

Para dar respuesta a este objetivo analizo los siguientes objetivos específicos:

Objetivo específico 1: Describir a la persona: madre y feto.

Objetivo específico .2: Describir la herencia.
Objetivo específico .3: Describir los cuidados maternos durante el embarazo

Objetivo específico 4: Formalizar qué repercusión tienen sobre el desarroIlo fetal el cuidado materno durante el embarazo

\section{MATERIAL Y MÉTODOS}

Para llevar a cabo el presente estudio de investigación, se ha empleado una metodología de tipo deductiva basada en la revisión bibliográfica, técnica de análisis de extracción del conocimiento y reunión de expertos. Las búsquedas bibliográficas han sido llevadas a cabo en las principales bases de datos Pubmed, Scielo y el metabuscador de la Biblioteca de la Universidad de Alcalá,

Se realizaron consultas on-line en el Diccionario de la Real Academia Española para la conceptualización en el desarrollo de las bases conceptuales, así como consultas en el B.O.E. para conocer la legislación vigente, la Declaración de Derechos Humanos y la OMS.

También fue necesario acudir a manuales de referencia en el ámbito de la Antropología humana, Genética, Herencia Humana, Bioética y Biología.

Para las búsquedas bibliográficas se emplearon las palabras clave: herencia, cultura, gestación, embarazo, cuida- 
dos, desarrollo prenatal y sus respectivos términos DeCS: genetics, culture, pregnancy, self care, embryology.

Además, se establecieron filtros por disponibilidad de texto, 5 años de publicación, idiomas (español e inglés) y ámbito de estudio (filosofía y antropología).

De dicha búsqueda, se seleccionaron 29 resultados por su relación con el tema de investigación.

A partir del análisis de la revisión bibliográfica, se elabora una tabla de relación de las recomendaciones de Autocuidado durante el embarazo con los Requisitos de Autocuidado Universal del Modelo de D.Orem.

Dicho estudio ha sido llevado a cabo durante la realización del Máster Universitario en Gestión y Aplicación del Conocimiento de Autocuidado en Enfermería, empleando un tiempo de estudio que abarca desde septiembre de 2017 hasta junio de 2018.

Se realizó educción con el grupo de expertos MISKC formado por: un Doctor en Ciencias de la Computación, dos Doctorandas, una Especialista en Enfermería Familiar y Comunitaria, y tres masters universitarios.

\section{RESULTADOS}

A continuación, se relatan los resultados de investigación.

\section{Resultado 1: Describir a la per-} sona

En lo que se refiere a la descripción de la persona, como ser vivo, tiene la capacidad de nacer, crecer, reproducirse y morir. (2) Como ser multidimensional: biológico, psíquico y social, llevará a cabo sus cuidados viéndose influenciado por el entorno y la cultura en la que se encuentre inmerso. (15)

Además, como especie correspondiente a los mamíferos, alberga a sus crías en el útero materno, haciendo de éste su entorno durante el desarrollo prenatal, siendo, por lo tanto, algo inherente al ser humano. (17)

Madre - hijo, durante el desarrollo prenatal, forman un conjunto en el que, el futuro recién nacido, es subconjunto materno. Ambos conforman un "sistema" en el que interaccionan de tal manera que, la madre supone el medio en el que el feto se desarrolla, dividiéndose en un "matroambiente", conformado por el útero materno, y un "microambiente", donde el feto se encuentra en contacto íntimo (placenta, cordón umbilical y otras membranas), y a través del 
cual recibe los nutrientes y aportes necesarios para su desarrollo. (18)

A su vez, el subconjunto materno, interacciona con el entorno, marcado por unas características culturales, ambientales, ideológicas... que determinará la manera en la que la mujer llevará a cabo sus cuidados, los cuáles influirán de manera positiva o negativa en el desarrollo fetal.

De tal manera que, aunque a priori, el "matroambiente" puede resultar una barrera que protege al feto del entorno y, en esencia, así lo es para determinados agentes ambientales, existen determinados factores teratogénicos capaces de atravesar la barrera placentaria y afectar al feto en mayor o menor medida en función de la etapa de desarrollo prenatal en la que se encuentra. (18)

Desde el marco de la Enfermería, el modelo de D. Orem, define a la perso- na como "una unidad con funciones bilógicas, simbólicas y sociales" centrándose en la capacidad que tienen éstas para llevar a cabo su autocuidado. Esta capacidad se verá afectada por unos factores internos y externos que denomina factores condicionantes básicos. (11)

En el caso de la mujer gestante, habremos de analizar sus FCB y los del feto. Para ello, se ha tenido en cuenta el análisis que realizó D. Antonio Quintero López en su trabajo de "Formalización de la transmisión del cuidado entre la madre y el feto durante la gestación," que realiza un análisis en una situación ideal de cuidado tanto para la madre como pare el feto. (17)

En este caso, se realizará un análisis de las diversas posibilidades con las que nos podemos encontrar: (Tabla 1)

Tabla 1. Análisis de FCB madre - feto en diversas posibilidades del cuidado (ideales / entorpecedoras). Elaboración propia. Fuente: (11)

\begin{tabular}{|c|c|c|}
\hline FCB & MADRE & FETO \\
\hline Edad & 18 - 45 años & $0-40 \pm 2$ semanas \\
\hline Estado de Dessarrollo & Adecuado a su edad & Adecuado a su edad gestacional \\
\hline Estado de Salud & Sana. Cuidados adecuados. & $\begin{array}{l}\text { Generalmente sano, } \\
\text { dependiente }\end{array}$ \\
\hline \multirow[t]{2}{*}{$\begin{array}{l}\text { Disponibilidad de } \\
\text { recursos }\end{array}$} & $\begin{array}{l}\text { Dispone de recursos } \\
\text { temporales }\end{array}$ & Dependiente de la madre \\
\hline & $\begin{array}{l}\text { Dispone de recursos } \\
\text { económicos }\end{array}$ & \\
\hline Factores del entrono & Entorno favorecedor & Dependiente de la madre \\
\hline Orientación sociocultura & Enculturizadas & Dependiente de la madre \\
\hline Patrones de Vida & Favorecedor para el cuidado & Dependiente de la madre \\
\hline $\begin{array}{l}\text { Sistema de apoyo } \\
\text { familiar }\end{array}$ & Favorables para el cuidado & Dependiente de la madre \\
\hline
\end{tabular}

\begin{tabular}{|c|c|c|}
\hline FCB & MADRE & FETO \\
\hline Edad & 18 - 45 años & $0-40 \pm 2$ semanas \\
\hline Estado de Dessarrollo & Adecuado a su edad & Adecuado a su edad gestacional \\
\hline Estado de Salud & Sana. Cuidados adecuados. & Generalmente sano, dependiente \\
\hline \multirow[t]{2}{*}{$\begin{array}{l}\text { Disponibilidad de } \\
\text { recursos }\end{array}$} & $\begin{array}{l}\text { Dispone de recursos } \\
\text { temporales }\end{array}$ & Dependiente de la madre \\
\hline & Limitación recursos económicos & Dependiente de la madre \\
\hline Factores del entrono & Entorno entorpecedor & Dependiente de la madre \\
\hline \multirow[t]{2}{*}{ Orientación sociocultural } & En proceso de enculturización & Dependiente de la madre \\
\hline & Aculturadas & \\
\hline Patrones de vida & Entorpecedor para el cuidado & Dependiente de la madre \\
\hline
\end{tabular}


Como su puede observar con el análisis de ambas tablas, tanto en una situación ideal de cuidado materno, entendiendo como tal aquella que provee un entorno favorecedor para el cuidado, contando con un adecuado sistema de apoyo familiar, así como como con unos patrones de vida favorecedores, como en una situación entorpecedora en la que la mujer gestante se puede encontrar en un entorno y unos patrones de vida que no favorecen sus cuidados, el feto es dependiente del cuidado materno.

Resultado 2: Describir la herencia

En cuanto al resultado Describir la herencia, se deduce que ésta se corresponde con los caracteres que son transmitidos por los progenitores a sus descendientes, pero ésta no puede ser entendida única y exclusivamente como una Herencia Biológica a la que solo afecta el genoma humano. En este sentido el ser humano como ser multidimensional adquiere de su entorno, a través de la cultura (dentro de la cual se encuentra la manera en la que llevan a cabo sus cuidados), una Herencia Cultural que le modifica y que se transmite a las siguientes generaciones.

Tal es así, que los cuidados que llevan a cabo a lo largo de su vida no sólo marcarán la suya propia, sino la de su descendencia, por lo que estos cuidados son capaces de modificar la propia estructura biológica del ser humano. (16)

\section{Resultado 3: Describir los cui-} dados maternos durante el embarazo

Para describir los cuidados maternos durante el embarazo, se deduce que, desde el marco teórico de Orem, la madre será agencia de autocuidado y agencia de cuidado dependiente del feto, de tal manera, que en función de cómo realice sus cuidados, éstos tendrán una repercusión sobre el feto. (11)

Lo ideal es una situación de Cuidar_me materno que vaya a favor de la supervivencia y del bienestar, de tal manera que repercutirá en un Cuidar_te "ideal" hacia el feto que favorecerá, a su vez, su supervivencia y bienestar. (14)

Dado que "el anticuidado forma parte de la realidad", una situación de anticuidando_me durante la gestación, conllevará a un anticuidando_te del feto.

Debido a la importancia que los cuidados maternos tienen durante el desarrollo prenatal, Organizaciones Internacionales de la Salud, como la OMS y organizaciones nacionales como la SEGO establecen una serie de recomendaciones de autocuidado para llevar a cabo un embarazo saludable. (19) 
Para el presente resultado, se ha tiende como las "acciones necesarias elaborado una tabla para clasificar dipara mantener los procesos vitales, la chas recomendaciones de autocuidado integridad de la estructura, funcionalidad con los Requisitos de Autocuidado Uniy bienestar". (11) versal de Orem (Tabla 2), los cuales en-

Figura 1 Correlación de la repercusión de los cuidados maternos durante el desarrollo prenatal. Fuente: Orem (1993). Creación propia.

Correlación de la repercusión sobre el desarrollo fetal de los cuidados maternos a través de los RAU

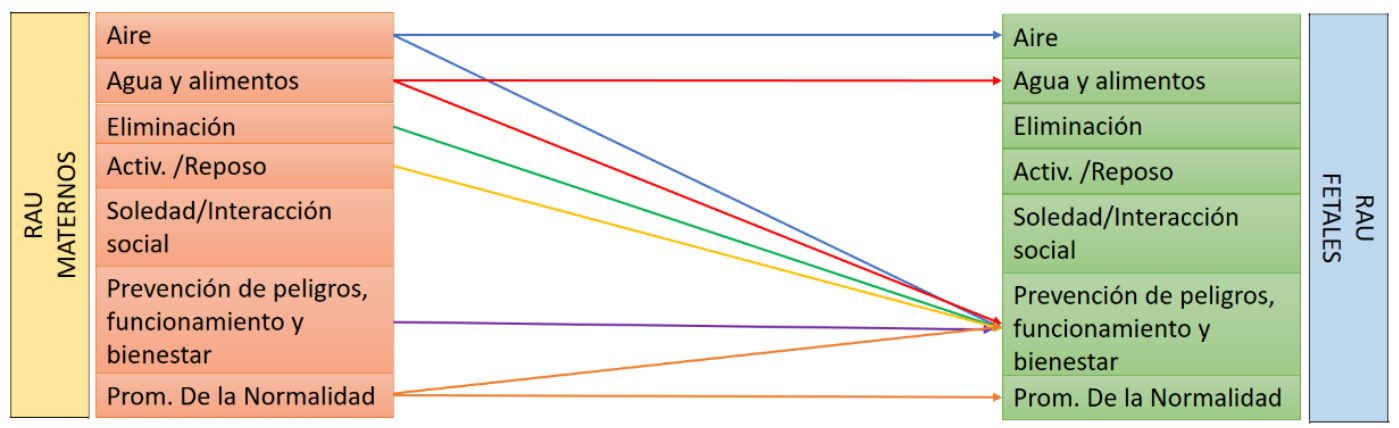

Resultado 4: Formalización de la repercusión que tienen sobre el desarrollo fetal los cuidados maternos durante el embarazo

Para poder formalizar la repercusión que tiene sobre el desarrollo fetal los cuidados maternos durante el embarazo, se realiza un análisis de los factores que afectan a la satisfacción de los Requisitos de Autocuidado Universal a través de los condicionantes de acción de Orem, de tal manera, que se pueda observar si, en el caso de que exista afectación materna, esto también supondría una afectación en el feto y, como resultado, se obtiene que se puede establecer una correlación en la que se ve claramente como los cuidados maternos tienen una repercusión sobre la el desarrollo en la etapa prenatal. (Figura 1)

De hecho, en su mayoría, afectarán al requisito de prevención de peligros, funcionamiento y bienestar, lo cual justificaría que dichos cuidados o anticuidados tienen una repercusión directa sobre el desarrollo prenatal.

\section{DISCUSIÓN}

La importancia de los cuidados maternos durante el embarazo es un hecho conocido por todos los profesionales de la salud, así como las consecuencias de éstos sobre el desarrollo fetal. 
Sin embargo, durante el presente estudio de investigación, se ha observado que no existen estudios que describan estos cuidados desde un Marco Teórico de Enfermería, ya que todos ellos tratan desde modelos biomédicos, haciendo referencia, más que a cuidados, a factores externos o internos a la madre con capacidad de atravesar la barrera placentaria (factores teratogénicos).

Únicamente, se ha encontrado un artículo, (17) que formaliza la existencia de una modificación de la Agencia de autocuidado, así como de la demanda de autocuidado, tanto de la madre como del feto, dada la existencia de una relación de dependencia entre ambos durante la gestación que, a su vez, se va modificando a lo largo de el mismo.

La no existencia de este tipo de estudio, así como, el hecho de que la disciplina enfermera es la responsable de la calidad de los cuidados de las personas hace que surja la necesidad de desarrollar este tipo de estudios.

\section{CONCLUSIÓN}

Tras haber llevado a cabo el estudio de las bases teóricas persona, cuidado y herencia, y haber realizado un análisis de la afectación de los RAU del Modelo de D. Orem, a través de los condicionantes de acción, del conjunto madre - feto, se puede establecer una co- rrelación entre los RAU que no se satisfacen en la madre y que esta insatisfacción afecta a la satisfacción de los RAU del feto.

De esta manera, tanto los RAU relacionados con Aporte suficiente de aire, agua y alimentos, eliminación, actividad y reposo, prevención de peligros y promoción de la normalidad afectarán fundamentalmente a la prevención de peligros y promoción de la normalidad del feto, lo cual puede explicar a través de un marco conceptual enfermero, la importancia de llevar a cabo unos cuidados prenatales adecuados. (11)

Por, lo tanto, puede decirse que, si la manera en la que la madre realiza su autocuidado afecta al feto, la herencia del cuidado se transmite y, de igual forma, si el anticuidado forma parte de la realidad, (14) podríamos concluir que la herencia del anticuidado se transmite.

\section{AGRADECIMIENTOS}

A los que, con su lucha, tesón y sus ganas de cambiar las cosas, se han puesto al frente del Máster Universitario en Gestión y Aplicación del Conocimiento del Autocuidado en Enfermería y me han enseñado a ver mi profesión sin la venda del sistema... GRACIAS, porque esta vuelta de tuerca me invita a pensar que sí se puede. 
A mi familia, a la propia y a la que

hemos creado, porque sin su apoyo no hubiera sido posible.

\section{BIBLIOGRAFÍA}

1. Diccionario de la RAE. [En línea] 2017. http:// dle.rae.es/.

2. Dadon, J. R.. Los seres vivos: características, origen, evolución. s.I. : Del Aula Taller, 2010.

3. Arteaga Martínez, Sebastián Manuel y García Peláez, María Isabel. Embriología humana y biología del desarrollo. Segunda. Ciudad de México : Médica Panamericana, 2017. pág. 6.

4. OMS. Nota descriptiva de Anomalías Congenitas de la Organización Mundial de la Salud. 2015.

5. Pellicer, A y Bonilla Musoles, F.M. Obstetricia y Ginecología. Para el grado de Medicina. Segunda . México : Ed. Médica Panamericana, 2014.

6. Santamaría G., J.M., Santamaría P., A. el Al. Bases filosóficas para el estudio del cuidado. Evolución histórica del pensamiento. Grupo MISCK. 2015.

7. Boyland, M. Aristoteles: Biology. The Internet Encyclopedia of Philosophy. [En línea] 2018. http:// www.iep.utm.edu.

8. Dignidad humana en Kant y Habermas. Michelini, D. J. 1, Julio de 2010, Revista anual de la Unidad de Historiografía e Historia de las Ideas - INCHUSA CONICET/Mendoza, Vol. 12. ISSN 1988 $348 \mathrm{X}$.

9. Fenomenología hermenéutica y sus implicaciones en enfermería. Ramírez, C. A. 1, Index de Enfermería, Vol. 25.

10. Hernández - Conesa, J.M., Moral de Calatrava, P. y Esteban - Albert, M. Fundamentos de Enfermería. Teoría y método. $2^{a}$ Edición corregida y ampliada. Madrid : Mc. Graw Hill/Interamericana de España S.A.U., 2003.
11. Orem, D. E. Modelo de Orem. Conceptos de enfermería en la práctica. Versión Española de la

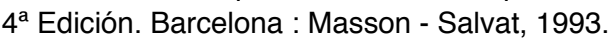

12. Alligood, M. R. Modelos y Teorías en Enfermería. Octava. Barcelona : Elsevier España, S.L., 2015.

13. Collière, M. Promover la vida. Madrid : Mc. Graw-Hill Interamericana, 1993.

14. Santamaría G, J.M. y Jímenez Rodríguez, M.L. Los Axiomas del Cuidado. Bases para su aprehensión conceptual. 1'a s.I. : Grupo MISCKC_ENE, 2016.

15. Beorlegui, C. Antropología filosófica. Dimensiones de la realidad humana. s.l. : Universidad de Deusto, 2017.

16. Ember, C.R. Antropología. $10^{\mathrm{a}}$. Madrid : Pearson, 2004.

17. Formalizacion de la transmisión del cuidado entre la madre y el feto durante la gestación. Quintero, L.A., González, A. 3, 2017, ENE. Revista de Enfermería, Vol. 11.

18. Hernández M., A., Lázaro, H., L y Polo, J., S. Enfermería del Recién Nacido Sano. Enfermería del niño y del adolescente II. Segunda edición. Madrid : Fuden, 2015.

19. SEGO. Recomendaciones gentileza de la SEGO y transmitidas por el Servicio de Obstetricia y ginecología del Hospital General universitario de Elche. 2010

20. Perry, S.E. y Cashion, K. Maternidad y salud de la mujer. Manual Clinico. Novena. Barcelona : Elsevier. Masson, 2008.

21. Formalización del equilibrio como motor del cuidado. Monsalvo San Macario, E. y Fernández Batalla, M. 3, 2017, ENE. Revista de enfermería, Vol. 11.

22. Síndrome de Abstinencia Neonatal. Evolución en los últimos diez años. Porcel Gálvez, A.M., Orteaga Martínez, S.M. y cols., y. 36, 2014, Enfermería Global, Vol. 13, págs. 337-351. 


\section{TABLAS Y ANEXOS}

Tabla 2. Recomendaciones de autocuidado durante el embarazo para satisfacer los RAU. Elaboración propia. Fuente: Orem (1993), OMS (2015) y SEGO (2010), (5), (20)

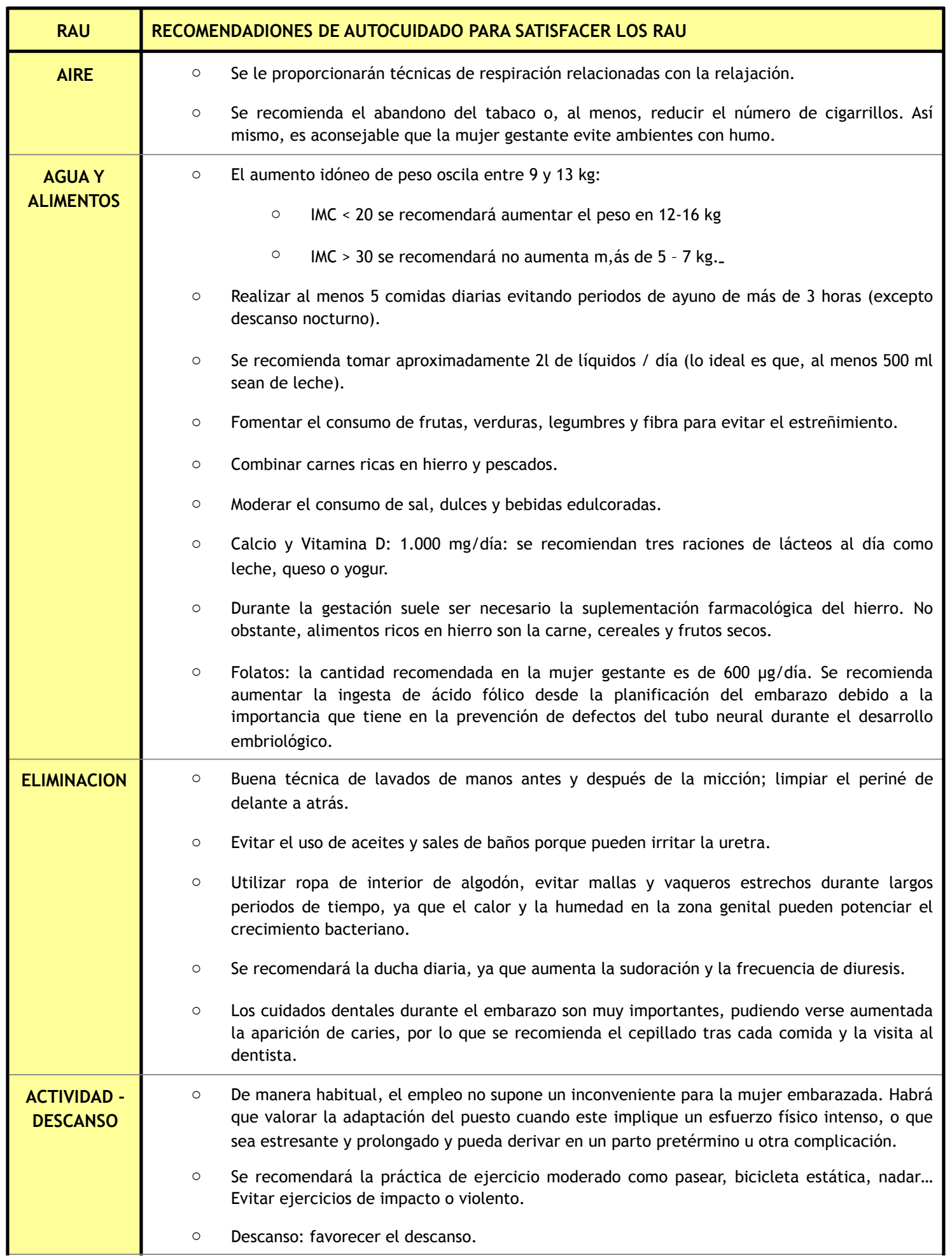




\begin{tabular}{|c|c|c|}
\hline \multirow[t]{4}{*}{$\begin{array}{l}\text { SOLEDAD - } \\
\text { INTERACCIÓN }\end{array}$} & $\circ$ & $\begin{array}{l}\text { Viajes: no está contraindicado viajar durante el embarazo. Sin embargo, las embarazadas de } \\
\text { alto riesgo deben evitar los viajes de largas distancias. }\end{array}$ \\
\hline & $\circ$ & $\begin{array}{l}\text { Se deben programar periodos de actividad y descanso durante el viaje y mantener una buena } \\
\text { hidratación. }\end{array}$ \\
\hline & $\circ$ & $\begin{array}{l}\text { Las vacunas para viajar a determinados países están contraindicadas durante el embarazo. } \\
\text { Si se viaje en coche se utilizarán cinturones de seguridad adaptados que con compriman el } \\
\text { abdomen materno y se programarán paradas para andar cada hora. }\end{array}$ \\
\hline & $\circ$ & $\begin{array}{l}\text { Si el viaje es en avión, deberá conocer las políticas de vuelo de la compañía, así como las } \\
\text { restricciones y recomendaciones. En su mayoría permiten el vuelo hasta las } 36 \text { semanas de } \\
\text { gestación en embarazos sin complicaciones. }\end{array}$ \\
\hline \multirow[t]{6}{*}{$\begin{array}{l}\text { PREV. } \\
\text { PELIGROS }\end{array}$} & $\circ$ & $\begin{array}{l}\text { Deben evitarse los contactos con animales no vacunados, posibles transmisores de infecciones } \\
\text { (toxoplasmosis, etc...) o con enfermos infectocontagiosos. }\end{array}$ \\
\hline & $\circ$ & El uso de medicamentos se realizará bajo prescripción médica. \\
\hline & $\circ$ & Se recomienda la abstinencia del consumo de alcohol y drogas de abuso. \\
\hline & $\circ$ & Se recomienda el abandono del tabaco o, al menos, reducir el número de cigarrillos. \\
\hline & $\circ$ & $\begin{array}{l}\text { Limitar el consumo de cafeína a 2-3 tazas de café, así como de bebidas que la contengan al } \\
\text { día. }\end{array}$ \\
\hline & $\circ$ & $\begin{array}{l}\text { Relaciones sexuales: los consejos de sexualidad consistirán en aclarar informaciones } \\
\text { erróneas, transmitir normalidad y, sugerir conductas alternativas. Ya que las ETS } \\
\text { (Enfermedades de Transmisión Sexual) pueden transmitirse a la madre y al feto, se } \\
\text { recomendará el uso de preservativo, si la mujer tiene riesgo de adquirirlas. }\end{array}$ \\
\hline \multirow[t]{5}{*}{ NORMALIDAD } & $\circ$ & $\begin{array}{l}\text { Apoyo psicosocial, estima, afecto, comprensión de las respuestas culturales y religiosas, } \\
\text { escucha. }\end{array}$ \\
\hline & $\circ$ & Se le enseñara a identificar las posibles complicaciones. \\
\hline & $\circ$ & Identificación del trabajo de parto pretérmino. \\
\hline & $\circ$ & Preparación para el parto. \\
\hline & $\circ$ & Preparación para la lactancia materna. \\
\hline
\end{tabular}

